\title{
Magda Donato: una pioniera del giornalismo spagnolo*
}

\author{
Margherita Bernard \\ Università degli Studi di Bergamo \\ margherita.bernard@unibg.it
}

https://dx.doi.org/10.12795/futhark.2013.i08.03

\begin{abstract}
Magda Donato occupies an important position within the frame of feminine journalism in the first decades of $X X$ century, but she deserves to be rescued from an unjust oblivion. This article studies Donato's collaboration with one of the most prestigious magazines of the time, España, founded and directed by the philosopher José Ortega y Gasset.Next to the most preeminent signatures of the culture of the time, Magda Donato's articles do not belong to an occasional collaboration; on the contrary, they constitute a column that faces the controversial themes of feminism, women'semancipation and family law. The journalist distinguishes herself by discoursive strategies which involve the reader and, at the same time, she produces a fragmentation of the point of view thanks to the use of a first person that doesn't always express her voice and her real ideas: sometimes it represents the prejudices that Donato wants to fight. By this tecnique, the writer pushes the reader (especially women) to think independently and critically about the themes that animated the debate of that time.

Keywords: journalism. feminine writing. emancipation.

Abstract: Nel panorama del giornalismo femminile dei primi decenni del Ventesimo secolo occupa un posto di rilievo la figura di Magda Donato, scrittrice ancora poco conosciuta che merita di essere riscattata da un ingiusto oblio. Questo lavoro esamina la collaborazione che la giornalista ebbe con una delle riviste culturali più prestigiose del periodo, España, fondata e diretta dal filosofo José Ortega y Gasset. Accanto alle firme più autorevoli della cultura dell'epoca, gli articoli di Magda Donato non si configurano come una collaborazione saltuaria, bensì costituiscono una rubrica che affronta i temi controversi del femminismo, dell'emancipazione della donna e della legislazione sulla famiglia. La giornalista si distingue attraverso strategie discorsive che coinvolgono il lettore e, allo stesso tempo, promuove una frammentazione del punto di vista grazie all'adozione di un io che non sempre coincide con la sua voce e con le sue reali idee, ma che talvolta incarna i pregiudizi che Donato intende combattere. Grazie a questa tecnica la scrittrice spinge il lettore, e in particolare le lettrici, a riflettere in modo autonomo e critico riguardo a temi che animavano il dibattito culturale dell'epoca.
\end{abstract}


Parole chiave: Giornalismo. Scrittura femminile. Emancipazionismo.

\section{Introduzione: donne e giornalismo nei primi decenni del Novecento}

È nota la quasi totale assenza delle donne nei manuali di storia del giornalismo spagnolo della prima metà del secolo $X X$; il numero limitato di presenze femminili, che senza dubbio corrisponde a una posizione marginale occupata dalla donna nelle redazioni dei giornali, non giustifica tuttavia l'omissione di figure che all'epoca ebbero un ruolo importante. Questo è un ambito di ricerca che può riservare molte sorprese e che potrebbe allungare il breve elenco di donne giornaliste nel quale, peraltro, si ripetono sempre i nomi illustri di quelle poche che arrivarono a esercitare un certo potere nel campo della carta stampata, come nel caso dell'antesignana Emilia de Pardo Bazán, seguita poi da Carmen de Burgos, Isabel Oyarzábal de Palencia, Sofía Casanova e poche altre ${ }^{1}$.

Negli anni che precedettero la Guerra Civile, furono diverse le scrittrici che collaborarono con la stampa e la loro azione non si limitò alle riviste dedicate al pubblico femminile; molte di loro si distinsero nei giornali e nelle riviste di attualità, di taglio generalista, a testimonianza dell'integrazione della donna nel ceto intellettuale europeo. La donna, che spesso era stata oggetto di dibattito nei primi decenni del secolo, cominciò ad assumere il ruolo di soggetto della riflessione politica, culturale e sociale.

Prenderò qui in considerazione il caso di un'importante rivista settimanale dei primi decenni del Novecento, España, che svolse un ruolo di grande rilievo nel panorama culturale del primo terzo del secolo XX. Questo periodico, creato nel 1915 da José Ortega y Gasset, rifletteva l'orientamento del suo fondatore, fermamente convinto che un cambiamento politico della nazione non sarebbe avvenuto senza avere alla base una trasformazione culturale. Questa posizione del filosofo spagnolo era condivisa da un gruppo di intellettuali e giovani universitari scon-

Questo studio è stato svolto nell'ambito del progetto "LA INCORPORACIÓN DE LAS ESCRITORAS ESPAÑOLAS A LOS MEDIOS DE PRENSA ENTRE 1868 Y 1936". Ministerio de Sanidad, Política Social e Igualdad. Instituto de la Mujer. $n^{\circ}$ Riferimento: 2011004-INV-00034.

1 Cfr. RoIg CAStellanos, Mercedes, La mujer y la prensa: desde el siglo XIX a nuestros días, Madrid, La Autora, D.L., 1977, pp. 57-89; PERINAT, Adolfo; MARRADES, María Isabel, Mujer, prensa y sociedad en España: 1800-1939, Madrid, Centro de Investigaciones Sociológicas, 1980.

Futhark 8 (2013)

Bernard, Magda Donato, 55-68

ISSN 1886-9300 
tenti della situazione politica del proprio paese ${ }^{2}$, che collaborarono a una pubblicazione che si proponeva la formazione di un senso critico nel pubblico lettore. I principi di ispirazione regeneracionista di questo "Settimanale della vita nazionale" sono espressi in modo esplicito nell'articolo di presentazione redatto dallo stesso Ortega:

"El desprestigio radical de todos los aparatos de la vida pública es el hecho soberano, el hecho máximo que envuelve nuestra existencia cotidiana. Todos sentimos que esa España oficial, dentro de la cual y bajo la cual vivimos, no es la España nuestra, sino una España de aluvión y de inepcia"3.

I collaboratori furono soprattutto giovani permeati dallo spirito orteghiano, ma parteciparono anche figure di rilievo del '98: Ramiro de Maeztu, Gregorio Martínez Sierra, Luis de Zulueta, Pío Baroja, Ramón Pérez de Ayala, Eugenio d'Ors e Juan Guixé, i quali, insieme al fondatore, formarono la prima redazione. Luis Araquistáin succedette a Ortega nel 1916 come direttore della rivista e infine, dopo un periodo sotto la guida di Manuel Azaña (con Cipriano de Rivas Cherif come vicedirettore), le pubblicazioni cessarono nel 1924.

Scorrendo l'indice dei collaboratori della prestigiosa rivista si possono notare i nomi più autorevoli del periodo ${ }^{4}$ nell'ambito della creazione letteraria e della riflessione filosofica. Non sorprende, se consideriamo le coordinate storiche e geografiche, che tra le più di trecento firme vi sia una quasi totale assenza di penne femminili. Le collaboratrici di España sono assai poche (non superano le dieci unità), molte di loro sono stra-

2 Cfr. Gómez ApARIClo, Pedro, Historia del periodismo español. De las guerras coloniales a la dictadura, Madrid, Editora Nacional, 1974, pp. 539-543; SÁNCHEZ ARANDA , J.J.; BARRERA, Carlos, Historia del periodismo español, Pamplona, Ediciones Universidad de Navarra, 1992, pp. 305-306; MORALES LeZCANO, Víctor, "Revista España, Semanario de la vida nacional (1915-1924)", Hispania. Revista española de historia, Vol. 39, N. 141, 1979, pp. 201-220. Per lo studio del giornalismo spagnolo dell'epoca ritengo imprescindibili i seguenti saggi: TUÑÓN DE LARA, Manuel, et.al. (ed.), Prensa y sociedad en España (1820-1936), Madrid, Cuadernos para el Diálogo, 1975; DESVOIS, Jean Michel, La prensa en España (19001931), Madrid, Siglo XXI, 1977; Azorín, Antonio, La hora de la pluma. Periodismo de la Dictadura y de la República, Madrid, Pre-Textos, 1987; FUENTES, Juan Francisco; Fernández Sebastián, Javier, Historia del periodismo español. Prensa política y opinión pública en la España contemporánea, Madrid, Síntesis, 1998; SÁlZ, Dolores; SeOANE,Ma. Cruz, Historia del periodismo en España, Madrid, Alianza Editorial, 1998.

3 España, Semanario de la vida acional, Año 1, N. 1, Madrid, 29 gennaio 1915, p. 1.

4 V. Morales Lezcano definisce giustamente la redazione della rivista come il quartier generale della generazione del 1914. Cfr. MORALES LeZCANO, Víctor, op. cit., p. 207. 
niere $^{5}$ e i loro articoli vengono pubblicati in modo sporadico. Tra le poche collaborazioni di autrici nazionali richiamano l'attenzione undici articoli firmati da Magda Donato, pubblicati tra il 21 agosto 1920 e il 5 febbraio 1921. Nove di essi (dal secondo al decimo) costituiscono una rubrica intitolata "Al margen del feminismo" in cui l'autrice affronta con notevole forza polemica i temi relativi all'emancipazione femminile che erano oggetto di accesi confronti nella società spagnola dell'epoca. Mi sembra importante sottolineare il fatto che per una sezione dedicata a argomenti che occupavano un posto centrale nel dibattito politico e culturale, sia stata chiamata una giovane giornalista, cosa che non costituiva certo una pratica abituale: gli articoli su temi come la posizione della donna nella società, il divorzio, il diritto familiare, che comparivano con notevole frequenza sulla stampa, erano, in maggioranza, scritti da uomini.

\section{Una giornalista all'avanguardia}

Magda Donato è lo pseudonimo di Carmen Eva Nelken: si tratta della sorella della più famosa Margarita Nelken ${ }^{6}$, che si sarebbe messa in

5 Le giornaliste o scrittrici che collaborarono con la rivista e che firmarono i testi con nomi e cognomi, non superano le dieci unità. Oltre a Magda Donato e a sua soreIla Margarita Nelken, ho potuto individuare: Beatriz Galindo (pseudonimo di Isabel de Oyarzábal de Palencia), Paula Jakoby, Ursula Johannsen, Ellen Key, Marina Lacort, Dulce Maria Loynaz, Fiona Mac Leod, Josefina de la Torre.

6 Magda Donato, giornalista, scrittrice e attrice, è una delle figure della prima metà del secolo XX che ancora non sono state riscattate completamente dall'oblio. In anni recenti sono stati pubblicati degli studi dedicati specialmente al suo lavoro come autrice di testi teatrali per l'infanzia. Cfr.: ENA BORDONADA, Angela, Novelas breves de escritoras españolas (1900-1936), Madrid, Castalia, 1990; NIEVA DE LA PAZ, Pilar, "Las autoras teatrales españolas frente al público y a la crítica (19181936)", AlH, Actas XI, Irvine, 1992, pp. 192-139; P NIEVA DE LA PAZ, Pilar, Autoras dramáticas españolas entre 1918 y 1936, Madrid, CSIC, 1993, pp. 255-262, NIEVA DE LA PAZ, Pilar, "Las escritoras españolas y el teatro infantil de preguerra", Revista de Literatura, 55, 1993, pp. 113-128; VILCHES, María Francisca; DOUGHERTY, Dru, La escena madrileña entre 1926 y 1931. Un lustro de transición, Madrid, Fundamentos, 1997, pp. 270-274; NIEVA DE LA PAZ, Pilar, "Revisando el cánon: hacia una selección crítica del teatro escrito por mujeres en la España de entreguerras" in ZAVALA, Iris M. (coord.), Breve historia feminista de la literatura española (en lengua castellana), Barcelona, Anthropos, 1998, pp. 155-184; BRAVO GUERREIRA, María Elena, "Para una recuperación del feminismo español. Recordando algunos nombres", Alaluz, Riverside, California, 1998, N. 1, pp. 73-82; RoDRIGO, Antonina, Mujer y exilio. 1939, Madrid, Compañía literaria, 1999, pp. 35-60; GARZÓN, Jacobo Israel; PUERTA de la, Javier "Carmen Eva Nelken (Magda Donato), esa desconocida", Raíces, Revista Judía de cultura, Año X, N. 43, 2000, pp. 43-50; VICENTE HeRNANDO de, César, "Estudio preliminar" a Magda Donato, Pipo, Futhark 8 (2013) Bernard, Magda Donato, 55-68 ISSN 1886-9300 
evidenza negli anni della Repubblica per la sua attività politica. Margarita Nelken fu una delle poche donne elette come deputate alle Corti Costituenti del 1931. Queste due intellettuali, nate in seno a una ricca famiglia ebrea di origine tedesca da parte di padre e francese da parte materna, avevano ricevuto un'educazione superiore, molto più moderna e liberale di quella che si impartiva solitamente alle giovani dell'epoca: parlavano perfettamente diverse lingue, avevano accesso a una cultura cosmopolita e spesso realizzavano viaggi all'estero. Erano entrambe destinate a far parte del gruppo di donne progressiste che negli anni Venti e Trenta contrastarono i modelli limitati che riservava loro la società dell'epoca.

Nonostante l'innegabile rivalità (che forse spinse Carmen Eva a scegliere uno pseudonimo che la distinguesse da Margarita) Magda Donato iniziò a frequentare, ancora adolescente, gli ambienti culturali progressisti in cui già si muoveva la sorella maggiore. Tornando agli aspetti di nostro interesse, Margarita Nelken era in contatto con i membri della redazione di España, con cui aveva collaborato nel 1916, pubblicando sei articoli su temi culturali e sociali ${ }^{7}$, ed è assai probabile che sia stata proprio lei a introdurre la sorella minore nella redazione della rivista.

Ad ogni modo, nonostante la giovane età -era nata nel 1898-, Magda Donato era già in possesso di un curriculum di tutto rispetto come giornalista. Nel 1917 la scrittrice, che aveva solo 19 anni, aveva iniziato una collaborazione con El Imparcial, il quotidiano della famiglia Gasset, nelle cui pagine divenne responsabile di una rubrica chiamata "Femeninas". Sotto questo titolo pubblicava cronache su temi che si ritenevano interessanti per il pubblico femminile: informazioni sulla moda, sui costumi sociali, sull'arredamento, sui bambini. La rubrica, che permise alla scrittrice di realizzare le prime esperienze in ambito giornalistico, appare

Pipa y el Lobo Tragalotodo-Pinocho en el país de los cuentos, Madrid, Publicaciones de la Asociación de Directores de Escena de España, 2000, pp. 9-39; HORMIGón, Juan Antonio (ed.), Autoras en la historia del teatro español (1500-2000), Madrid, Publicaciones de la Asociación de Directores de Escena de España, 1996-2000, pp. 913-927; MANGINI, Shirley, Las modernas de Madrid, Barcelona, Península, 2001, pp. 188-189; González SANTAMERA, Felicidad, "El teatro femenino" in HUERTA CALVo, Javier, Historia del teatro español, Madrid, Gredos, 2003, Tomo II, pp. 2503-2525; SOTOMAYOR SÁEZ, María Victoria, "Una vida para el teatro", Lazarillo: revista de la Asociación de amigos del libro infantil y juvenil, Vol. 10, 2003, pp. 78-83; BERNARD, Margherita, "Magda Donato y la promoción del trabajo femenino" in BERNARD Margherita; ROTA Ivana (eds.), En prensa.Escritoras y periodistas en España (1900-1939), Bergamo, Sestante, 2010, pp. 109-134. Cfr. anche DONATO, Magda, Reportajes, Edizione e introduzione di Margherita Bernard, Sevilla, Renacimiento, 2009 y DonATO, Magda, Cómo vive la mujer en España, Edizione e introduzione di Margherita Bernard, Sevilla, ArCiBel, 2009.

7 Gli articoli furono pubblicati nei numeri 57, 60, 77, 79, 81 e 85 di España dal 24 febbraio 1916 fino al 7 settembre dello stesso anno.

Futhark 8 (2013)

Bernard, Magda Donato, 55-68

ISSN 1886-9300 
in linea con il ruolo che tradizionalmente era riservato alle donne nelle redazioni. Questi primi articoli, che dimostrano senza alcun dubbio un talento straordinario, risultano particolarmente interessanti perché sono marcati da alcuni tratti stilistici che diverranno una costante nella carriera della nostra scrittrice, tanto dal punto di vista giornalistico quanto di quello letterario.

Lo stile di Magda Donato si contraddistingue per l'adozione di un atteggiamento comunicativo che cerca di stabilire un dialogo con il pubblico lettore. Emergono il linguaggio colloquiale, le domande retoriche e, soprattutto, la presenza reiterata dell'io della scrittrice, che si propone costantemente come interlocutore. Leggendo questi articoli, il lettore aveva -e ha tuttora- l'impressione di partecipare a una conversazione brillante, ironica, a volte francamente umoristica, sempre di un livello culturale elevato. L'atteggiamento di Magda Donato è caratterizzato dall'ironia e dall'umorismo con i quali osserva i temi che tratta di volta in volta e, grazie a questo taglio, riesce a mettere in discussione gli argomenti sui quali si dibatte. Come esempio, possiamo leggere una cronaca in cui commenta scherzosamente le consuetudini che regolano i fidanzamenti in Spagna. Attraverso il trattamento stilistico emerge la posizione della giovane scrittrice che mette in ridicolo una società legata a riti sociali che considera superati:

"En España por ejemplo, la mujer tiene que gustar de golpe y porrazo a un hombre que pasa a su lado por la calle; luego tienen los dos que aprender el abecedario de los sordomudos, y si el noviazgo empieza en invierno tienen que exponerse: ella a una pulmonía permaneciendo horas enteras en el balcón, él a un tortícoli por tener otras tantas horas la cabeza levantada para contemplar el rostro de su reciente bien amada" 8 .

I temi frivoli di molti di questi articoli si prestano a un trattamento ironico e spiritoso che tuttavia non era quello adottato dalla maggioranza delle giornaliste nelle cronache di questo tipo. L'umorismo della scrittrice attribuisce una distanza critica a testi apparentemente superficiali ${ }^{9}$ : Attraverso lo stile ironico, fa filtrare la critica dei costumi dell'epoca che le sem-

"Femeninas", Hay mil maneras de casarse, El Imparcial, 3 de abril 1917, p. 3.

Inoltre, non possiamo dimenticare che nel 1917 Magda Donato aveva già cominciato una tormentata relazione con Salvador Bartolozzi, noto illustratore, scenografo e autore di testi infantili, che sarebbe poi diventato il compagno di tutta una vita. Bartolozzi, egli stesso grande umorista, frequentava ambienti in cui si alternavano scrittori molto interessati al linguaggio umoristico, come Arniches, Muñoz Seca, Jardiel Poncela, Antoniorrobles e Ramón Gómez de la Serna: non possiamo escludere l'ipotesi per cui l'attitudine ironica e burlesca adottata dallo scrittore nelle sue creazioni abbia esercitato un'influenza sulla giovane compagna.

Futhark 8 (2013)

Bernard, Magda Donato, 55-68

ISSN 1886-9300 
brano assurdi e promuove un modello di sano scetticismo rispetto a quanto socialmente accettato $^{10}$, con l'intenzione evidente di modificare la mentalità conservatrice dei lettori, creando un'opinione più moderna e più in sintonia con l'evoluzione della società europea. II contenuto di questi articoli si evolve con il passare del tempo, e in effetti i temi trattati iniziano a riguardare questioni meno superficiali, relative al ruolo della donna nella società, l'emancipazione e il lavoro femminile, che erano alcuni tra i principali interessi della scrittrice. E' doveroso segnalare che l'uso dell'umorismo è un tratto che caratterizza la sua singolare personalità; in effetti l'umorismo era considerato all'epoca una peculiarità maschile e veniva praticato assai di rado dalle scrittrici. Tuttavia, il talento umoristico di Donato era qualcosa di riconosciuto all'epoca della sua collaborazione con España. II direttore teatrale Cipriano de Rivas Cherif, elogiando l'impegno della scrittrice nel progetto del Teatro de la Escuela Nueva, sottolinea il suo "talento literario, de tan varonil humorismo"11. A questa caratteristica di assoluta modernità se ne aggiungono altre che definiscono Magda Donato come una giornalista precorritrice dei nuovi tempi.

Negli articoli pubblicati su El Imparcial, Magda Donato difende talvolta l'idea che al giornalista competa esclusivamente informare, diffondere idee. Eppure molto presto la sua scrittura si allontana dal giornalismo asettico, puramente informativo, pur ammettendo che anche quella possa essere una strada percorribile. Nella sua visione, il giornalista deve creare opinione, giungere alle coscienze con il proposito di contribuire alla costruzione di una società migliore. In un testo pubblicato nel 1918 , osserva:

"Pero el verdadero periodismo, el periodismo verdaderamente periodístico es el de la información. [...] Pero el periodismo práctico debe tener algo más que la parte pintoresca: su deber es hacer mucho bien, destruir lo que está mal y ayudar a la edificación de lo que pudiera estar bien"12.

10 La radicata postura ironica e umoristica di Magda Donato si percepisce in modo evidente quando, nel 1928, inizia a collaborare con la rivista Estampa, fondata di recente e in cui viene pubblicata una sezione di moda intitolata "Eva. Páginas de la mujer". Dal numero 1 sino al 39 si alternano diverse firme (Lucie de Max, Marguerite Choineau, María Teresa Fontanar) che scrivono i testi di tema femminile; dal numero 40 (2 ottobre 1928) Magda Donato si incarica di questa sezione. Gli articoli firmati dalla nostra scrittrice cambiano in modo drastico il tono serio, neutro ed esclusivamente informativo di quelli precedenti, tornando a essere ironici e amabilmente scherzosi.

11 Rivas Cherif, Cipriano de, "El Teatro de la Escuela Nueva", La Pluma, Año II, N 2, Madrid, 1921, p. 242.

12 DONATO, Magda, "Femeninas", "La mujer y el periodismo", El Imparcial, 13 gennaio 1918, p. 3.

Futhark 8 (2013)

Bernard, Magda Donato, 55-68

ISSN 1886-9300 
Fedele alla propria impostazione progressista, Magda Donato pratica per tutta la vita un giornalismo incentrato su temi e situazioni che conosce approfonditamente e che presenta al lettore in modo critico, come abbiamo visto, adottando talvolta l'ironia come ponte comunicativo diretto con il pubblico.

\section{La collaborazione con España: strategie comunicative}

Se ora andiamo a esaminare gli articoli pubblicati su España, possiamo osservare che lo stile della scrittrice definisce una personalità giornalistica che si è già affermata nel settore e che gode di successo tra i lettori: l'individualità dell'autrice, nonostante la giovane età, si è imposta con sicurezza in un ambiente di certo poco favorevole alle donne. Nel primo articolo che Magda Donato pubblica su España il 21 agosto 1920 -“ "Por no enterarse' dice..."- la scrittrice si scaglia con ironia e brillante disinvoltura contro gli uomini che scrivono sul femminismo senza sapere affatto di che si tratta e senza fare lo sforzo di documentarsi correttamente. II sarcasmo della scrittrice rifiuta le impostazioni teoriche: dopo aver indicato l'unico giornalista che secondo lei scrive sul tema con più cognizione rispetto a molte donne -Luis de Zulueta, noto educatore e redattore di España-, segnala nomi e cognomi dei rappresentanti della categoria degli "inofensivos", che si limitano a riproporre vecchi cliché, e dei "pedantes", additando Cristóbal de Castro ${ }^{13}$ come rappresentante di questa categoria e concentrando il proprio attacco su di lui. La scrittrice accusa questo giornalista di non informarsi, di parlare con "serena inconsciencia de cosas que desconoce en absoluto", di riempire i suoi articoli di luoghi comuni. II tono del testo, inizialmente, è frivolo, discorsivo e ironico; la scrittrice parla di Cristóbal de Castro in terza persona. Più avanti si rivolge a lui direttamente, apostrofandolo con gran forza polemica e con un atteggiamento sarcastico. II consiglio finale di Magda Donato al suo collega riassume un giudizio netto e inappellabile:

"Como usted comprenderá, señor Cristóbal de Castro, yo no he tenido intenciones de darle a usted una lección; usted es sin duda un hombre de mucho talento y un escritor extraordinario; pero créame usted, señor Cristóbal de Castro, hable usted de toros, o de agricultura, o de lo que usted quiera, pero

13 CAStRo GutiÉRREZ, Cristóbal de (1874-1953) fu uno scrittore prolifico, traduttore, giornalista e politico. Nelle sue narrazioni si dimostrò un difensore dei diritti della donna. Ricevette gli elogi di Concha Espina, Emilia de Pardo Bazán e Carmen de Burgos. Affrontò tematiche femministe nei romanzi Mujeres, Eva moderna, Mujeres extraordinarias.

Futhark 8 (2013)

Bernard, Magda Donato, 55-68

ISSN 1886-9300 
no de feminismo. Porque hay muchos hombres, señor Cristóbal de Castro, a quienes les pasa lo que a muchas mujeres: que debían dedicarse en este mundo a las labores propias de su sexo"14.

La scrittrice assume un atteggiamento che la definisce come una vera precorritrice del moderno giornalismo d'opinione ${ }^{15}$ : la rilevanza assegnata al punto di vista del giornalista, l'esperienza personale presentata ed elaborata in modo critico, il tono marcatamente colloquiale che cerca un contatto diretto con il pubblico sono elementi che implicano il proposito di influenzare l'opinione dei lettori adottando strategie retoriche che mettano in risalto l'efficacia comunicativa. La scrittrice si rivolge direttamente al pubblico attraverso formule o domande retoriche -"Recuerdan ustedes el cuento aquel..."; “¿Creerán ustedes que ha necesitado para ello informarse profundamente?"; " ¿Y de qué está enterado el señor Cristóbal de Castro?"- che trasformano il lettore in interlocutore di un dialogo con l'autrice e, più avanti, nell'ultima parte del testo, in un testimone delle critiche dirette a Castro, che la Donato rimprovera, apostrofandolo direttamente.

L'uso di un linguaggio colloquiale -“iqué sé yo!”; “¡Quiá! Nada de eso."; "los que se meten a resolver problemas que no conocen ni por el forro"; "... acaban por forzar la siempre bobalicona admiración de las masas"- non implica affatto che la scrittrice adotti uno stile trascurato: la struttura dell'articolo, l'uso degli strumenti retorici, la progressione dall'ironia al sarcasmo denotano uno straordinario controllo del discorso e una grande accuratezza letteraria che ha come obiettivo quello di portare il pubblico verso una riflessione critica.

L'ultimo aspetto che mi preme segnalare è la presenza costante dell'io della scrittrice, che presenta in primo luogo il proprio punto di vista e la propria reazione emotiva -"he leído crónicas"; "me ha sorprendido"; "me alegré"; "he oído decir"; "iqué sé yo!"- e che poi difende esplicitamente il suo modo serio e professionale di concepire il giornalismo e la cronaca, in contrapposizione ironica con la superficialità del cronista criticato $^{16}$. II discorso veemente di Magda Donato fa leva sull'abile uso

14 “'Por no enterarse' dice...”, España, N. 277, 21 agosto 1920, p. 5. Tutte le citazioni nel testo si trovano nella stessa pagina.

15 Su questo tema cfr.: GROHMAnN, Alexis; SteEnmeIJeR, Maarten (ed.), El columnismo de escritores españoles (1975-2005), Madrid, Verbum, 2006 e MONTESA PEYDRO, Salvador (ed.), Literatura y periodismo: la prensa como espacio creativo, Málaga, Publicaciones del Congreso de Literatura Española, 2003.

16 "Frente a pruebas tan concluyentes, no me queda más remedio que callarme, yo, pobre infeliz, no iniciada en la omnisciencia del señor Cristóbal de Castro, yo humilde cronista, que para enterarse me he limitado a ir a Ginebra, hablar con la presidenta de la "Alianza internacional", leer con mis propios ojos una carta tan in- 
del linguaggio che le permette di lasciar filtrare nel testo la presenza del suo io soggettivo (come donna femminista) e del suo io testimoniale (come cronista responsabile) ${ }^{17}$.

\section{La frammentazione dell'io}

In seguito a questo esordio brillante, i successivi articoli pubblicati su España (tranne l'ultimo) compongono, come già è stato detto, una rubrica intitolata "Al margen del feminismo". Nonostante il tema abbia un forte legame con il primo testo, ci troviamo di fronte a una modalità discorsiva completamente differente e, da un certo punto di vista, sconcertante. Nei dieci articoli pubblicati, la scrittrice compie una riflessione autocritica sul femminismo, commentando con il suo abituale sarcasmo gli errori che, secondo lei, commettono le femministe spagnole e i conflitti che si sono scatenati tra le diverse organizzazioni, oppure si lancia in una battaglia ironica in difesa delle posizioni del conservatorismo borghese più radicale, con il chiaro scopo di smontare i suoi argomenti; le sue strategie comunicative si avvalgono di una dislocazione del punto di vista basata su una sorta di frammentazione dell'io dell'autrice.

In "Una junta"18 Magda Donato adotta il modello della cronaca e descrive, raccontando in prima persona e con grande sfoggio di brillante ironia, una riunione del direttivo di un gruppo femminista. Risulta evidente il desiderio di stigmatizzare le debolezze, la mancanza di senso della realtà e l'inconsistenza delle proposte delle partecipanti e delle loro rivendicazioni. La scrittrice descrive abitudini sociali di poca rilevanza, come la mancanza di puntualità o la superficialità di alcuni atteggiamenti, ma mostra anche con chiarezza la pericolosa frammentazione del movimento femminista spagnolo e il conformismo di molte delle sue rappresentanti. Magda Donato presenta se stessa come una delle partecipanti alla riunione e assume in prima persona tutti gli atteggiamenti, a volte francamente ridicoli, che racconta, ottenendo un effetto di distanziamento ironico e talvolta umoristico, senza manifestare una critica diretta. L'atteggiamento dell'“io personaggio" aderisce in modo assoluto alle affermazioni e alle istanze delle protagoniste del resoconto, le quali non

teresante como significativa [...]."DONATO,Magda " 'Por no enterarse' dice...", España, N. 277, 21 agosto 1920, p. 5.

17 Cfr. EnA BordonadA, Angela, "Las crónicas del primer tercio del siglo XX: entre el yo testimonial y el yo subjetivo", El Compás de las letras, Vol. 1, 1992, pp. 228243

18 "Al margen del feminismo. Una junta", España, N. 280, 11 septiembre 1920, Año VI, pp. 11-12.

Futhark 8 (2013)

Bernard, Magda Donato, 55-68

ISSN 1886-9300 
possono non sembrare risibili ai lettori. I temi discussi nella riunione mettono in rilievo l'opinione negativa della scrittrice verso una determinata maniera di affrontare e concepire il femminismo, nascondendo questo giudizio sotto un'apparente condivisione. La misura che alla fine della riunione approvano le donne presenti è un esempio dell'atteggiamento caustico della scrittrice:

"Y entonces se levanta una señora y anuncia que [...] se celebrará un gran mitin para protestar contra la escasez de bancos de madera en los parques públicos de Madrid. ¿No le parece a la honorable sociedad[...] que sería de todo punto necesario y provechoso que la "Agrupación de feministas españolas" estuviese representada en este mitin? ¡Bravo! El entusiasmo es general. La proposición es aprobada por unanimidad". (p. 12)

La critica indiretta è più sferzante proprio in virtù dell' io fittizio rappresentato dalla scrittrice che mostra gli aspetti assurdi e ridicoli di certo femminismo come se li condividesse. L'articolo si conclude con queste parole:

"Y nos separamos con el pecho henchido por el entusiasmo y la conciencia satisfecha por la convicción de haber contribuido una vez más a la realización de dos grandes ideales: el triunfo de la causa feminista y la regeneración del país". (p. 12)

L'io autobiografico del primo articolo commentato stabiliva il punto di vista reale da cui la scrittrice osservava gli avvenimenti e le permetteva di esprimere le sue vere opinioni; a partire da questo secondo articolo, invece, ci troviamo dinnanzi a un io fittizio, letterario; la scrittrice si sdoppia assumendo un'ottica -quella di un femminismo iberico conservatore, borghese e classista- che ha l'intenzione di criticare e in questo modo mette in rilievo -talvolta in maniera iperbolica- i suoi limiti e gli errori che commette. Grazie a questo distanziamento retorico, la scrittrice vuole promuovere una riflessione attiva, che trae origine, più che dall'argomentazione, dall'ironia e dall'esasperazione parodica; il suo obiettivo è portare le lettrici a considerare gli errori che nella sua opinione il femminismo spagnolo sta commettendo e promuovere un ragionamento libero da pregiudizi.

Adottando questa linea di sdoppiamento, Magda Donato sembra approvare la sfiducia e il disprezzo nascosto che le femministe spagnole hanno dimostrato nel Congresso di Ginevra ${ }^{19}$ verso i paesi dell'Europa del Nord che, invece, nella sua reale opinione ammira incondizionatamente, ritenendo che questi stati rappresentino l'avanguardia sul tema

19 Nel 1920 si celebrò il VII Congresso della IWSA (International Women's Suffrage Alliance). II primo progetto di organizzazione del Congresso a Madrid fu abbandonato e la riunione si realizzò nel giugno del 1920 a Ginevra. Cfr. FAGOAGA, Concha, La voz y el voto de las mujeres (1877-1931), Barcelona, Icaria, 1985, pp.158 y ss. 
dell'emancipazione femminile. La scrittrice vuole mettere in guardia le femministe del suo paese dai rischi di una posizione puramente ideale e speculativa -al contrario Donato ritiene indispensabile prendere misure concrete che possano favorire le condizioni di vita delle donne- e lo fa difendendo ironicamente le posizioni che vuole sanzionare e che le sembrano pericolose per i fini che il movimento femminista si propone:

"Nuestra labor no es por lo tanto material; no por eso deja de existir, al contrario. [...] La diferencia esencial [con respecto al feminismo de los países del Norte] es que allí todo va encaminado hacia lo práctico, lo prosaicamente práctico; mientras que nuestros trabajos son más bien de propaganda ideal, espiritual.

"Mi reino no es de este mundo", dijo Cristo. El nuestro tampoco"20.

L'ammirazione che la scrittrice nutriva verso il femminismo dei paesi nordici affonda indubbiamente le radici nella formazione laica e cosmopolita che aveva ricevuto dalla famiglia, formazione che la portò ad avere un atteggiamento critico verso l'associazionismo femminista prudente, borghese e cattolico come quello che pochi anni dopo -nel 1926avrebbe dato luogo alla creazione del Lyceum Club di Madrid

II sarcasmo della scrittrice giunge alla propria massima espressione nell'articolo intitolato “ ¿El divorcio? ¡Jamás!", in cui affronta un problema che preoccupava importanti settori della società. II tema era di grande attualità e di evidente rilevanza sociale: le pagine di España costituivano una tribuna privilegiata per il prestigio di cui godeva la rivista. II punto di vista adottato dalla giornalista è quello di una donna conservatrice che difende tutti i luoghi comuni relativi all'istituzione matrimoniale, raffigurando in questo modo un quadro realista di buona parte della popolazione spagnola e facendo risaltare l'ipocrisia dei conservatori e di molte donne. II tono apocalittico con cui inizia il testo sottolinea con ironia la durezza della battaglia che aveva scatenato il tema del divorzio:

"¡Españolas! Un gran peligro amenaza la tranquilidad de nuestra vida, la paz de nuestros hogares, la seguridad del pan nuestro de cada día. Soplan vientos nefandos; se avecina una discordia más temible que la ola roja [...]. En una palabra, hermanas mías, nos amenaza la implantación de la ley de divorcio"21.

20 "Al margen del feminismo. La conspiración de los países latinos", España, N. 283, 2 ottobre 1920, pp.10-11.

21 "Al margen del feminismo.¿El divorcio? ¡Jamás!”, España, N. 284, 9 ottobre 1920, pp.15-16.

Futhark 8 (2013)

Bernard, Magda Donato, 55-68

ISSN 1886-9300 
Osservando modalità discorsive così peculiari sorge legittimamente una domanda: questo tipo di comunicazione ha colto nel segno? Le lettrici hanno percepito il distacco ironico voluto dalla scrittrice? Effettivamente, il sarcasmo della giornalista e lo sdoppiamento retorico del'io che ho segnalato non furono ben recepiti da tutte le lettrici della rivista, alcune delle quali, stando a quanto si afferma nell'articolo "Hablando en serio", pubblicato nel mese di novembre ${ }^{22}$, scrissero a Magda Donato accusandola di screditare il femminismo: "se me acusa de tomar en broma cosas que son muy serias". Ciò dimostra che l'interazione ricercata con le lettrici aveva avuto successo, nonostante il metodo applicato dalla scrittrice non avesse riscosso sempre una buona accoglienza tra il pubblico che sembra sconcertato di fronte alle affermazioni contenute nell'articolo. La scrittrice rifiuta le critiche e si difende affermando la sua assoluta fedeltà alla realtà dei fatti e negando ogni intenzione ironica: rifiuta di assumere il suo io reale e rivendica la totale sincerità del'io fittizio. Successivamente, si lamenta di aver ricevuto critiche per il suo articolo contro il divorzio:

"Una vez, una sola, me han tomado en serio: cuando hace poco escribí un artículo contra el divorcio recibí tres cartas de señoras muy indignadas porque yo fuera tan timorata y retrógrada" 23 .

Come difesa, la scrittrice adotta un atteggiamento di falsa modestia, confessando che le sue poche risorse le impediscono di esprimersi in modo più chiaro, profondo e originale come sanno fare le donne che hanno scritto gli statuti della Asociación Nacional de Mujeres Españo$\operatorname{las}^{24}$. E qui Magda Donato non perde l'opportunità di lanciare una frec-

22 "Al margen del feminismo. Hablando en serio", España, 27 novembre 1920, p. 14.

23 Le opinioni di Magda Donato rispetto all'istituzione matrimoniale e al divorzio erano indubbiamente avanzate rispetto all'epoca. Nell'intervista di Artemio Precioso, che possiamo leggere come prologo nell'edizione del suo romanzo breve $\mathrm{La}$ carabina, si dichiara contraria al divorzio per la semplice ragione che è fermamente contraria al matrimonio, considerato una "institución [...] inmoral" e che ritiene "destinada a desaparecer". La scrittrice teme che il divorzio "suavizando el matrimonio" ritardi la sua abolizione. Inoltre si dichiara favorevole all'amore libero che, secondo lei, garantisce maggiore sincerità e pertanto è moralmente più elevato dell'istituzione matrimoniale che ha come conseguenza la falsità e l'ipocrisia. Cfr. PRECIOSo, Artemio, "A manera de prólogo" in Magda Donato, La carabina, La Novela de Hoy, Madrid, Rivadeneyra, 1924, p. 6.

24 La ANME (Asociación Nacional de Mujeres Españolas), costituita nel 1918, era un'organizzazione moderata che rifuggiva gli estremismi di destra e sinistra, la cui leader principale, María Espinosa, difendeva un programma che si proponeva di ottenere per le donne diritti civili e politici. Nello stesso periodo veniva fondata anche la UME (Unión Mujeres de España); questa organizzazione si collocava a sinistra della ANME. Nel direttivo della UME spiccava María de la O Lejárraga. Magda Donato militava in questa organizzazione più radicale e più orientata al socialismo. Negli anni Venti tra i due raggruppamenti si librò una vera battaglia sulla questione di chi dovesse rappresentare la Spagna alla IWSA (International Wo- 
ciata alla più importante, ma anche più conservatrice delle associazioni femministe dell'epoca. La frase che cita come esempio di pensieri elevati è un concentrato di banalità retoriche: - "Si te preguntan qué es feminismo, contesta: redención de la mujer, perfección de las sociedades, elevación de la humanidad" - e su questa affermazione si applica il sarcasmo feroce della scrittrice:

"Y ya está; con una frase solamente ya queda usted enterado, lector, de lo que es el feminismo, y ya posee una clave infalible para hacérselo comprender de un modo definitivo a su interlocutor por muy cerril e ignorante que sea"25.

men's Suffrage Alliance). Negli articoli di Magda Donato è inevitabile percepire gli echi di questa polemica. Cfr. FAGOAGA, Concha, op. cit., pp. 128-165.

25 "Al margen del feminismo. Hablando en serio", cit., p. 14.

Futhark 8 (2013)

Bernard, Magda Donato, 55-68

ISSN 1886-9300 